\title{
Allele-specific polymerase chain reaction for the discrimination of elite Korean cattle associated with high beef quality and quantity
}

\author{
Wonhee Lee ${ }^{1, \star}$, Insik Nam ${ }^{3, \star}$, Daehyun Kim ${ }^{4}$, Kukdong Kim ${ }^{5}$, and Yoonseok Lee ${ }^{1,2}$ \\ ${ }^{1}$ Department of Biotechnology, Hankyong National University, Gyeonggi-Do, \\ Anseong, 17579, Republic of Korea \\ ${ }^{2}$ Center for Genetic Information, Hankyong National University, Gyeonggi-Do, \\ Anseong, 17579, Republic of Korea \\ ${ }^{3}$ Department of Animal Life Convergence Science, Hankyong National University, \\ Gyeonggi-Do, Anseong, 17579, Republic of Korea \\ ${ }^{4}$ Livestock Research Institute, Gyeongsangbuk-Do, Yeongju, 36052, Republic of Korea \\ ${ }^{5}$ Ministry of SMEs and Startups, Chungcheongnam-Do, Chunan, 31169, Republic of Korea \\ * These authors contributed equally to this work. \\ Correspondence: Yoonseok Lee (yoonseok95@hknu.ac.kr)
}

Received: 22 January 2021 - Revised: 18 August 2021 - Accepted: 29 November 2021 - Published: 7 February 2022

\begin{abstract}
Techniques such as direct sequencing and PCR-RFLP (restriction fragment length polymorphism) are widely used to analyze the genotypes of livestock. However, these conventional methods have the disadvantage of taking a lot of time and incurring considerable cost. The allele-specific PCR method performs PCR using two primers, and a single nucleotide polymorphism (SNP) genotype can be identified through electrophoresis, saving time and cost. Highly accurate results can be obtained by designing specific primers according to the allele of the SNP under study, utilizing primer binding to a complementary matching sequence. In this study, we established a genotyping system with the AS-PCR technique, using SNPs related to the improvement of the meat quality and meat mass of Korean cattle. Using the PRIMER1 program, we designed specific primers for SNPs located at the $3^{\prime}$ end, with one SNP marker in the HSPBI gene related to meat quantity and two SNP markers in the $A D H I C$ and $F A S N$ genes related to meat quality in cattle. AS-PCR was performed on 10 Korean cattle using the primers designed with this system, and the genotypes could be identified by the size of the PCR product amplified as a result of electrophoresis. In the case of the HSPB1 g.2352T $>\mathrm{C}$ SNP, the T allele was amplified to $148 \mathrm{bp}$, and the $\mathrm{C}$ allele was amplified to $222 \mathrm{bp}$. The $A D H 1 C$ c.-64T $>\mathrm{C}$ SNP was amplified to $492 \mathrm{bp}$ at the $\mathrm{T}$ allele and $330 \mathrm{bp}$ at the $\mathrm{C}$ allele. The FASN g.17924G > A SNP A allele was amplified to $377 \mathrm{bp}$ and the $\mathrm{G}$ allele to $507 \mathrm{bp}$. The results for each SNP genotype were verified using direct sequencing, which showed that the genotypes identified by direct sequencing and the genotypes identified by the AS-PCR method matched exactly. The AS-PCR method therefore appears to be valuable for use in a genotyping system.
\end{abstract}




\section{Introduction}

Recently, research into the development of DNA markers related to economic traits such as meat quantity and quality in cattle has been reported. Among these DNA markers, single nucleotide polymorphisms (SNPs) are single base differences between the DNA of different individuals. SNPs are more suited for use as genotyping markers than conventional markers such as restriction fragment length polymorphisms (RFLPs), amplified fragment length polymorphisms (AFLPs), and simple sequence repeats (SSRs), because SNPs are the most abundant and stable form of genetic variation in the cattle genome. With new developments in biotechnology, SNPs are becoming favored genetic markers for use in marker-assisted selection breeding programs. In a field farm, traditional SNP genotyping methods such as DNA sequencing and PCR-RFLP, have been used to select elite cattle with excellent meat quality and quantity. Usually, these methods are expensive, time-consuming, and laborintensive and require specialized equipment. There is therefore a need for simple and accurate genotyping assays that can be implemented in laboratories lacking access to sophisticated equipment. Recently, allele-specific PCR (AS-PCR) has been widely used for low-throughput applications in genetic breeding in cattle (Zhao et al., 2019; Darawi et al., 2013). AS-PCR is based on the extension of primers, which only occurs when the $3^{\prime}$ end is a perfect complement to the template. In principle, SNPs can be detected using allelespecific PCR primers based on the 3'-terminal nucleotide of a primer that corresponds to a specific SNP site. However, reliable discrimination between alleles cannot be achieved using this method. To overcome this problem, allele-specific primers with an additional base pair change within the three bases closest to the SNP site between have been used (Cha et al., 1992; Kwok et al., 1994). Considerable research has been conducted using SNPs identified as being related to meat quality and quantity. Zhang et al. (2014) published a study that demonstrated that the expression of the heat-shock protein beta-1 (HSPB1) gene induces muscle formation during the growth of beef cattle (Zhang et al., 2014), and a study comparing variations in the weight of Korean cattle according to the SNP genotype of the HSPBI gene was published. Kim et al. (2009) reported that the FASN (g.17924 G > A) gene is closely associated with fatty acid metabolism, controlling the quality of Korean beef. SNPs can be used as DNA markers for producing high-quality Korean beef as discriminating meat-related genetic factors (Kim et al., 2009). Using the c.-64T > C SNP of the ADH1C gene, Ward et al. (2012) reported that marbling in cattle with the TT genotype of the $A D H 1 C$ gene was higher than that in TC and CC genotypes during 5 months of restriction of vitamin A in Angus crossbred steers (Ward et al., 2012). Peng et al. (2017) analyzed the association of the c.-64T $>$ C SNP of the $A D H 1 C$ gene with intramuscular fat deposition in Korean cattle during experiments with vitamin-A restriction in Korean cattle steers
(Peng et al., 2017). The aim of this study was to establish a genotyping system based on AS-PCR analysis using three SNPs in the $H S P B 1, A D H 1 C$, and FASN genes, to make genotyping more convenient and to reduce the amount of labor and time required.

\section{Materials and methods}

\subsection{DNA preparation}

Genomic DNA was extracted from 10 sirloin tissues using G-DEX ${ }^{\mathrm{TM}}$ Genomic DNA extraction kits (Intronbio, South Korea). First, $100 \mathrm{mg}$ of Korean beef tissue was added to a $2 \mathrm{~mL}$ tube, with $600 \mu \mathrm{L}$ of lysis buffer and $0.5 \mu \mathrm{L}$ each of proteinase $\mathrm{K}$ and RNase. The lysis process was carried out at $37^{\circ} \mathrm{C}$ overnight. The following day, $300 \mu \mathrm{L}$ of PPT buffer was added and vortexed, the solution was centrifuged at $12000 \mathrm{rpm}$ for $10 \mathrm{~min}$, and the supernatant was transferred to a $1.5 \mathrm{~mL}$ tube. Then $300 \mu \mathrm{L}$ of isopropanol was added to the supernatant, which was mixed and stored at $-20^{\circ} \mathrm{C}$ for $1 \mathrm{~h}$. The mixture was then centrifuged at $12000 \mathrm{rpm}$ for 5 min to obtain the DNA pellet, which was washed with $99 \%$ ethanol. The genomic DNA was suspended in $100 \mu \mathrm{L}$ of TE buffer after the ethanol dried and was stored at $-20^{\circ} \mathrm{C}$ for use in this study. The sequences of the extracted DNA were confirmed using electrophoresis.

\subsection{Primer design and amplification of AS-PCR}

In order to conduct AS-PCR for each SNP in the HSPBI (g.2352T > C), ADH1C (c.-64T > C), FASN (g.17924G > A) genes, primers were designed using PRIMER (http:// primer1.soton.ac.uk, last access: 15 November 2019). FASTA sequences extracted from Ensemble (http://www. ensembl.org, last access: 15 November 2019) were used. The position of the SNP was marked in the PRIMER1 program so that SNP was located at the $3^{\prime}$ end of the specific primer. To increase the specificity of the primer, the third nucleotide from the $3^{\prime}$ end of the specific primer was mismatched. The primers used in this study are shown in Table 1. When ASPCR was performed using the primer for each gene, the PCR products were amplified to $148 \mathrm{bp}$ for the T allele, $222 \mathrm{bp}$ for the $\mathrm{C}$ allele and $319 \mathrm{bp}$ for the control in the HSPBI gene. For the $A D H 1 C$ gene, the T allele was amplified to $492 \mathrm{bp}$, the $\mathrm{C}$ allele to $330 \mathrm{bp}$, and the control to $762 \mathrm{bp}$. For the FASN gene, the A allele was amplified to $377 \mathrm{bp}$, the $\mathrm{G}$ allele to $507 \mathrm{bp}$, and the control to $830 \mathrm{bp}$. For the AS-PCR mixture used in this study, Hotstart Taq DNA polymerase kits (BIONEER, South Korea), primer mixture, DNA template, and sterilized distilled water were added to make a total reaction volume of $50 \mu \mathrm{L}$. The primer mixture was added at the concentrations shown in Table 1. As shown in Table 2, the AS-PCR cycling used in this study was performed with a pre-denaturation step at $95^{\circ} \mathrm{C}$ for $15 \mathrm{~min}$, followed by 35 cycles of amplification; denaturation at $95^{\circ} \mathrm{C}$ for $30 \mathrm{~s}$, an- 


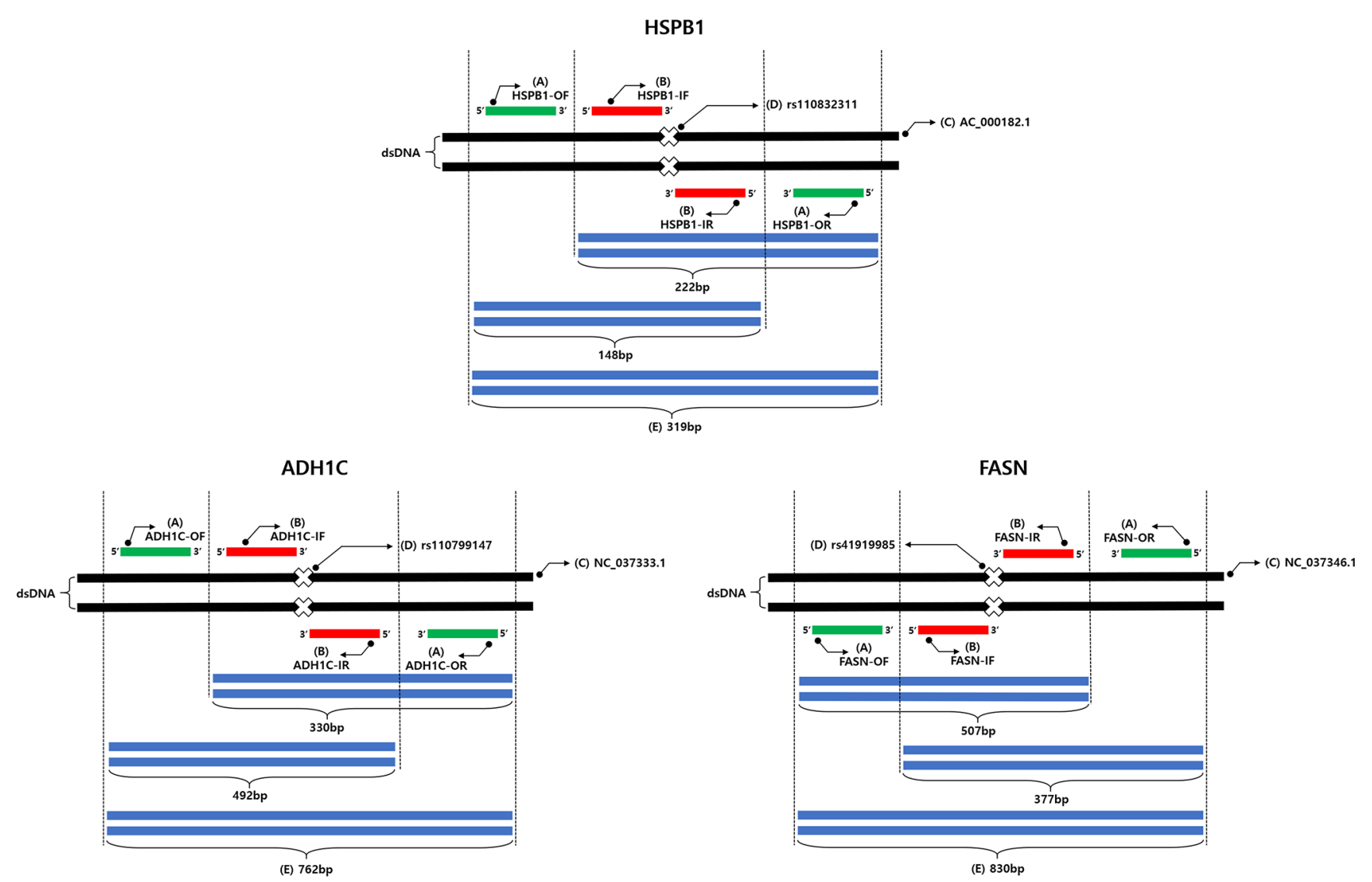

Figure 1. Schematic diagram of AS-PCR amplification and size of fragments. (A) Outer primers are indicated by green rectangles. (B) Inner primers are indicated by red rectangles. (C) DNA sequences were retrieved from NCBI using their accession numbers. (D) The rs numbers of SNPs are indicated by cross marks, and the amplified size of each SNP genotype is indicated by blue rectangles. (E) Amplicon of positive control.

nealing for $30 \mathrm{~s}$, and extension at $72^{\circ} \mathrm{C}$ for $1 \mathrm{~min}$. The final extension was performed at $72{ }^{\circ} \mathrm{C}$ for $5 \mathrm{~min}$. The annealing temperatures were different for $H S P B 1, A D H 1 C$, and FASN as follows: $\mathrm{HSPB} 163^{\circ} \mathrm{C}, A D H 1 \mathrm{C} 61^{\circ} \mathrm{C}$, and $F A S N 61^{\circ} \mathrm{C}$. After AS-PCR, electrophoresis was performed at $100 \mathrm{~V}$ for 40 min in $0.5 \times$ tris-borate-EDTA buffer on $1.5 \%$ agarose gels stained with ethidium bromide.

\subsection{DNA sequencing for the validation of genotyping}

DNA sequencing was carried out to verify the AS-PCR results. The PCR product used for sequencing was amplified using a pair of outer primers for AS-PCR, and Labopass ${ }^{\mathrm{TM}}$ IP pro-Taq DNA polymerase (Cosmogenetech, South Korea). Sequencing was performed using an AllInOneCyclerTM 384 well PCR system (BIONEER, South Korea) using BigDye Terminator v3.1 sequencing kits (Applied Biosystems, USA), and then sequenced using an ABI3730XL (Applied Biosystems, USA).

\section{Results and discussion}

\subsection{Association of identified SNPs with beef quality and quantity in Korean cattle}

In this study, we used three SNPs identified by a previous study into the production of beef of high quality and quantity in Korean cattle. The SNPs were related to the production of high-quantity beef in Hanwoo. The g.2352T > C SNP in the $H S P B 1$ gene produced a significant difference in meat quantity, and the performance of animals with the CC homozygous genotype was higher than that of other genotypes (Suh et al., 2020). In a study into the production of high-quality beef, the CT heterozygote genotype of c.-64T > C SNP in the $A D H 1 C$ gene was found to have an increased marbling score compared with the other genotypes (Peng et al., 2017). Oh et al. (2012) suggested that the marbling score of animals with the GG homozygous genotype of the g.17924G > A SNP in the FASN gene was significantly increased over those of other genotypes.

In order to analyze the genotype of these SNPs, methods such as PCR-RFLP and direct sequencing are widely used. 
(a)

(b)
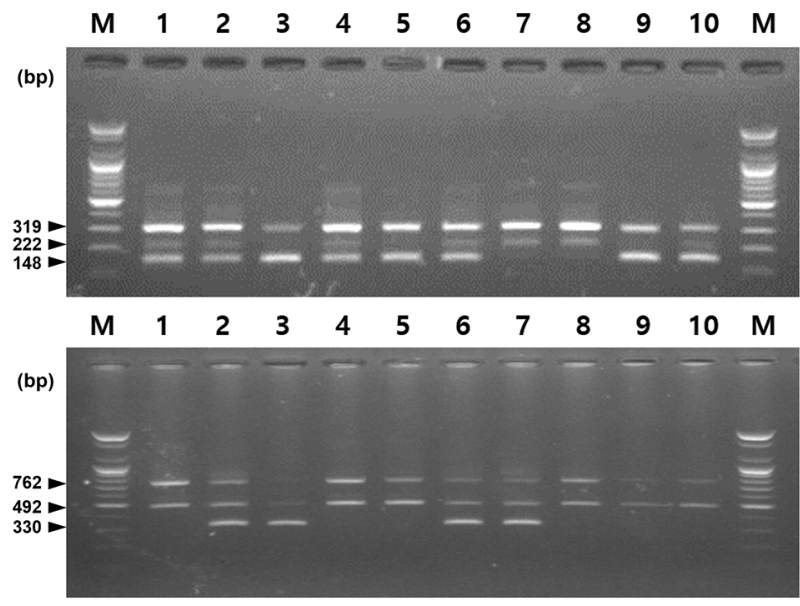

(c)

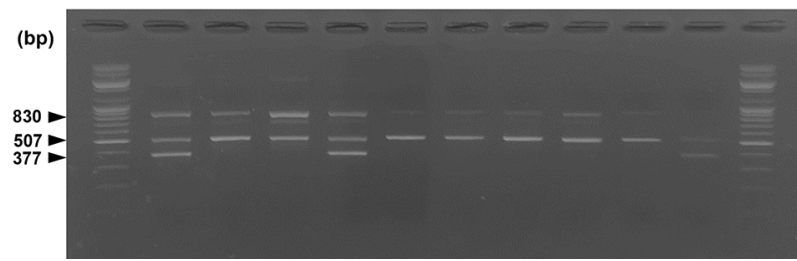

(d)
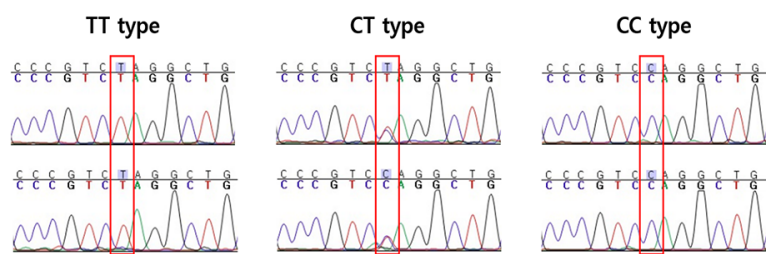

(e)

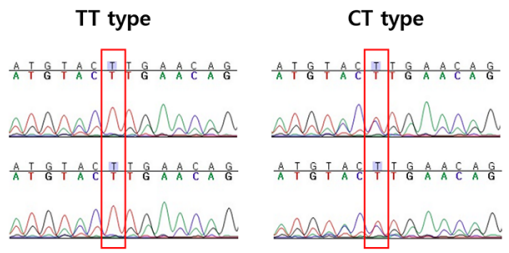

Figure 2. Results of electrophoresis for each genotype amplified using three SNPs. M: molecular weight marker. (a) HSPB1 g.2352T > C SNP, CC type was amplified to 222 and $319 \mathrm{bp}$, CT type was amplified to 148, 222, and $319 \mathrm{bp}$, TT type was amplified to 148 and $319 \mathrm{bp}$; (b) $A D H 1 C$ c.-64T > C SNP, TT type was amplified 492, 762 bp, CT type was amplified 330, 492, 762 bp; (c) FASN g.17924G > A SNP, GG type was amplified 507, $830 \mathrm{bp}$, AG type was amplified 377, 507, $830 \mathrm{bp}$; (d) result of direct sequencing for the HSPB1 g.2352T > C SNP; (e) result of direct sequencing for the $A D H 1 C$ c.-64T $>$ C SNP.

Table 1. Information, sequences, and concentrations of primers for SNPs used in this study.

\begin{tabular}{|c|c|c|c|c|}
\hline Gene & $\begin{array}{c}\text { SNP } \\
\text { (dbSNP no.) }\end{array}$ & Primer name & Primer sequence $\left(5-3^{\prime}\right)$ & $\begin{array}{l}\text { Concentration of primer } \\
\text { in AS-PCR (uM) }\end{array}$ \\
\hline \multirow{4}{*}{ HSPB 1} & \multirow{4}{*}{$\begin{array}{c}\text { g. } 2352 \mathrm{~T}>\mathrm{C} \\
(\mathrm{rs} 110832311)\end{array}$} & HSPB1-OF & GCACGGCTACATTTCCCGTTGCTTCAC & 1.2 \\
\hline & & HSPB1-OR & TTACTTGTTTTCCGGCTGTTCGGACTTCCC & 1.2 \\
\hline & & HSPB1-IF & TAACTCTCGTCTACCCTCTTTGCCCGGCC ${ }^{*}$ & 0.6 \\
\hline & & HSPB1-IR & GTCCACACCGGGGGGCAGCATA* ${ }^{*}$ & 0.6 \\
\hline \multirow{4}{*}{ ADH1C } & \multirow{4}{*}{$\begin{array}{c}\text { c.-64T }>C \\
(\text { rs } 110799147)\end{array}$} & ADH1C-OF & ACTGGTGTCTGATTTCTCTGTTGTGAAG & 0.5 \\
\hline & & ADH1C-OR & AGAATTCCAGTTGAGCTATTCCAGATCC & 0.5 \\
\hline & & ADH1C-IF & AATCTGTGCAATCTATCTCTTGTATGTCCC ${ }^{*}$ & 0.5 \\
\hline & & ADH1C-IR & TTACAGACTTACAGGCTCTTCCCTGTTAAي* & 0.5 \\
\hline \multirow{4}{*}{ FASN } & \multirow{4}{*}{$\begin{array}{l}\text { g. } 17924 \mathrm{G}>\mathrm{A} \\
\text { (rs41919985) }\end{array}$} & FASN-OF & GGGAAATCCGGCAGCTCACAATCCACAA & 0.8 \\
\hline & & FASN-OR & GTGTAGGCCATCACGAAGGTGTGCGAGC & 0.8 \\
\hline & & FASN-IF & CACCACCGTGTTCCACAGCCTGGACA* ${ }^{*}$ & 0.2 \\
\hline & & FASN-IR & GGCCATAGGTGGGGATGCTGAGCTTTGC & 0.2 \\
\hline
\end{tabular}

* Underlined means site of SNP.

However, these methods have several disadvantages. The experimental process is time-consuming and expensive, and the enzyme treatment generates analytical errors. We proposed an allele-specific polymerase chain reaction (AS-PCR), for analyzing genotypes without the need for enzyme treatment, to overcome these disadvantages.

\subsection{AS-PCR for HSPB1 g.2352T > C, ADH1C c. $-64 \mathrm{~T}>\mathrm{C}$ and FASN g.17924G $>\mathrm{A}$}

In this study, we performed AS-PCR genotyping analysis using three SNPs HSPB1 g.2352T > C, ADHIC c.-64T > C, and FASN g.17924G > A known to be related to beef quantity and quality. AS-PCR is a PCR-based method that uses specif- 
Table 2. Different sizes of amplicons according to use of paired primers for different SNPs.

\begin{tabular}{|c|c|c|c|c|c|}
\hline \multirow{2}{*}{$\begin{array}{l}\text { SNP } \\
\text { (dbSNP no.) }\end{array}$} & \multirow[t]{2}{*}{ Genotype } & \multirow[t]{2}{*}{ Assay } & \multicolumn{2}{|c|}{ Inter acting primers } & \multirow[t]{2}{*}{ Size of fragment (bp) } \\
\hline & & & Forward & Reverse & \\
\hline \multirow{10}{*}{$\begin{array}{l}\text { g. } 2352 \mathrm{~T}>\mathrm{C} \\
(\mathrm{rs} 110832311)\end{array}$} & \multirow{3}{*}{$\mathrm{CC}$} & \multirow{3}{*}{ Variant type } & HSPB1-OF & \multirow{3}{*}{ HSBPB1-OR } & 319 \\
\hline & & & & & \\
\hline & & & HSPB1-IF & & 222 \\
\hline & \multirow{4}{*}{$\mathrm{CT}$} & \multirow{4}{*}{ Wild type and variant type } & HSPB1-OF & \multirow{3}{*}{ HSPB1-OR } & 319 \\
\hline & & & & & \\
\hline & & & HSPB1-IF & & 222 \\
\hline & & & HSPB1-OF & HSPB1-IR & 148 \\
\hline & \multirow{3}{*}{$\mathrm{TT}$} & \multirow{3}{*}{ Wild type } & \multirow{3}{*}{ HSPB1-OF } & HSPB1-OR & 319 \\
\hline & & & & & \\
\hline & & & & HSPB1-IR & 148 \\
\hline \multirow{9}{*}{$\begin{array}{l}\text { c. }-64 \mathrm{~T}>\mathrm{C} \\
(\mathrm{rs} 110799147)\end{array}$} & \multirow{3}{*}{$\mathrm{CC}$} & \multirow{3}{*}{ Wild type } & ADH1C-OF & \multirow{3}{*}{ ADH1C-OR } & 762 \\
\hline & & & & & \\
\hline & & & ADH1C-IF & & 330 \\
\hline & \multirow{3}{*}{$\mathrm{CT}$} & \multirow{3}{*}{ Wild type and variant type } & ADH1C-OF & \multirow[b]{2}{*}{ ADH1C-OR } & 762 \\
\hline & & & ADH1C-IF & & 330 \\
\hline & & & ADH1C-OF & ADH1C-IR & 492 \\
\hline & \multirow{3}{*}{$\mathrm{TT}$} & \multirow{3}{*}{ Variant type } & \multirow{3}{*}{ ADH1C-OF } & ADH1C-OR & 762 \\
\hline & & & & & \\
\hline & & & & ADH1C-IR & 492 \\
\hline \multirow{9}{*}{$\begin{array}{l}\text { g. } 17924 G>A \\
(r s 41919985)\end{array}$} & \multirow{3}{*}{ AA } & \multirow{3}{*}{ Wild type } & FASN-OF & \multirow{3}{*}{ FASN-OR } & 830 \\
\hline & & & & & \\
\hline & & & FASN-IF & & 377 \\
\hline & \multirow{3}{*}{$\mathrm{AG}$} & \multirow{3}{*}{ Wild type and variant type } & FASN-OF & & 830 \\
\hline & & & FASN-IF & FASN-OR & 377 \\
\hline & & & FASN-OF & FASN-IR & 507 \\
\hline & & & & FASN-OR & 830 \\
\hline & GG & Variant type & FASN-OF & & \\
\hline & & & & FASN-IR & 507 \\
\hline
\end{tabular}

ically designed primers to permit amplification by DNA polymerase only if the nucleotide at the $3^{\prime}$ end of the primer perfectly binds to one complementary base in the variant or wild-type sequences. The expected fragment sizes following amplification using a primer pair designed for specific SNPs in the previous study are shown in Fig. 2. As shown in Fig. 2, two primer pairs were used in this study: the outer primer and the inner primer. In order to design the outer and inner primer for each SNP, DNA fragments which contained flanking DNA sequences of $1000 \mathrm{bp}$ towards the 5 and $3^{\prime}$ directions from the SNP position were used. The sequence was designed to mismatch at the third base at the $3^{\prime}$ end of the inner primer, to increase the specificity for the alleles of the three SNPs (Liu et al., 2012). As shown in Table 1, the third base at the $3^{\prime}$ end of the inner primer was mismatched from $\mathrm{T}$ to $\mathrm{G}(H S P B 1)$, A to $\mathrm{C}(A D H 1 C)$, and $\mathrm{C}$ to $\mathrm{A}(F A S N)$.

In the first-round PCR, the amplified fragment using the outer primer of these SNPs was used as a positive control, and subsequently the fragment was distinguished by size, depending on the combination of outer and inner primer in the second-round PCR. The concentration of primer affected the amplification when using primer combinations in the ASPCR method. The primer concentrations of the three SNPs used in this study are shown in Table 1.

The different sizes of fragments based on the combination of outer and inner primers are shown in Table 2 and Fig. 2. For the g. $2352 \mathrm{~T}>\mathrm{C}$ SNP of HSPB1 gene, the fragment size of the positive control was $319 \mathrm{bp}$, amplified by HSPB1-OF/HSPB1-OR. Based on the positive control, the 
HSPB1-IF/HSPB1-OR and HSPB1-OF/HSPB1-IR combinations amplified the $\mathrm{C}$ and $\mathrm{T}$ alleles to 222 and $148 \mathrm{bp}$, respectively. Therefore, as shown in Fig. 2, the g.2352T $>\mathrm{C}$ SNP of the HSPBI gene was found to be 148 and $319 \mathrm{bp}$ in the TT homozygous genotype and 222 and $319 \mathrm{bp}$ in the $\mathrm{CC}$ homozygous genotype. However, in the CT heterozygous genotype, all of the alleles for these SNPs $(148,222$, and $319 \mathrm{bp}$ ) were amplified.

For the c.-64T $>$ C SNP of the ADHIC gene, the positive control amplicon was $762 \mathrm{bp}$, when amplified by the ADH1C-OF/ADH1C-OR combination. Based on the positive control amplicon, the ADH1C-IF/ADH1C-OR and ADH1C-OF/ADH1C-IR combinations amplified the $\mathrm{C}$ and $\mathrm{T}$ allele to 330 and $492 \mathrm{bp}$, respectively. The c.-64T > C SNP of the $A D H 1 C$ gene was identified as 330 and $492 \mathrm{bp}$ alleles, amplified to 762 and $492 \mathrm{bp}$ in the TT homozygous genotype, 762 and $330 \mathrm{bp}$ in the CC homozygous genotype, and 330, 492, and 762 bp in the CT heterozygous genotype (Fig. 2b). Finally, in the g.17924G > A SNP of the FASN gene, FASNIF/FASN-OR, and FASN-OF/FASN-IR amplified the A and $\mathrm{G}$ alleles to 377 and $507 \mathrm{bp}$ based on the $830 \mathrm{bp}$ amplicon. As shown in Fig. 2c, the A (377 bp) and G (507 bp) alleles were amplified to 507 and 830 bp in the GG homozygous genotype, 377 and $830 \mathrm{bp}$ in the AA homozygous genotype, and 377,507 , and $830 \mathrm{bp}$ in the AG heterozygous genotype.

Direct sequencing was performed to validate the AS-PCR results. The genotype predicted by the AS-PCR and the genotype confirmed through direct sequencing were consistent (Fig. 2d and e).

\section{Conclusions}

Currently, PCR-RFLP and the sequencing method, which are the most widely used for genotyping, use expensive chemical reagents such as restriction enzymes and fluorescent nucleic acid stains, also require professional labor and equipment, and are time-consuming due to complicated experimental process. Therefore, there is a need for a simple and accurate genotyping method that can be implemented in an environment without professional labor and sophisticated equipment. The AS-PCR method developed in this study requires only basic equipment: a thermal cycler and an electrophoresis system. Also it is cost-effective, because it uses only DNA polymerase and does not use restriction enzymes or fluorescent nucleic acid stains, and high sensitivity and specificity is provided by the inclusion of positive and negative controls according to principle that primers bind only to complementary sequences. This research team analyzed 3 SNPs related to meat quantity and quality in Korean cattle using AS-PCR method, and all 10 samples were amplified to match the expected product size according to the designed primers, achieving successful experimental results. Therefore, the use of the AS-PCR method will enable researchers to carry out genotyping analysis for SNPs (HSPB1 g.2352T $>\mathrm{C}$,
$A D H 1 C$ c.-64T $>$ C, FASN g. $17924 \mathrm{G}<$ A) without the use of sophisticated instrumentation and at low cost.

Data availability. The original data of the paper are available from the corresponding author upon request.

Author contributions. WL, DK, KK, and YL conceived and designed the experiment. WL and YL performed and analyzed the experiment. WL and IN wrote the paper. All authors reviewed and approved the final paper.

Competing interests. The contact author has declared that neither they nor their co-authors have any competing interests.

Disclaimer. Publisher's note: Copernicus Publications remains neutral with regard to jurisdictional claims in published maps and institutional affiliations.

Acknowledgements. This work was supported by a research grant from the Rural Development Administration, Republic of Korea.

Financial support. This research has been supported by the NextGeneration BioGreen 21 Program (grant no. PJ01332201), Rural Development Administration, Republic of Korea.

Review statement. This paper was edited by Steffen Maak and reviewed by Seunghwan Lee, Memis Ozdemir, and one anonymous referee.

\section{References}

Cha, R. S., Zarbl, H., Keohavong, P., and Thilly, W. G.: Mismatch amplification mutation assay (MAMA): application to the c-H-ras gene, PCR Meth. Appl., 2, 14-20, https://doi.org/10.1101/gr.2.1.14, 1992.

Darawi, M. N., Ai-Vyrn, C., Ramasamy, K., Hua, P. P. J., Pin, T. M., Kamaruzzaman, S. B., and Majeed, A. B. A.: Allele-specific polymerase chain reaction for the detection of Alzheimer's disease-related single nucleotide polymorphisms, BMC Med. Genet., 14, 27, https://doi.org/10.1186/1471-2350-14-27, 2013.

Kim, K., Kim, N., Kim, S., Lee, S., and Lee, J.: Single Nucleotide Polymorphic Marker in Hanwoo FASN Gene for Distinction of Beef Quality and Method for Determining the Hanwoo Beef Quality Using the Same SNP Marker, KR101158475B1, available at: https://patents.google.com/patent/KR101158475B1/ko (last access: 21 January 2022), 2009.

Kwok, S., Chang, S. Y., Sninsky, J. J., and Wang, A.: A guide to the design and use of mismatched and degenerate primers, PCR 
Meth. Appl., 3, S39-S47, https://doi.org/10.1101/gr.3.4.S39, 1994.

Liu, J., Huang, S., Sun, M., Liu, S., Liu, Y., Wang, W., Zhang, X., Wang, H., and Hua, W.: An improved allele-specific PCR primer design method for SNP marker analysis and its application, Plant Methods, 8, 34, https://doi.org/10.1186/1746-4811-8-34, 2012.

Oh, D., Lee, Y., La, B., Yeo, J., Chung, E., Kim, Y., and Lee, C.: Fatty acid composition of beef is associated with exonic nucleotide variants of the gene encoding FASN, Mol. Biol. Rep., 39, 4083-4090, https://doi.org/10.1007/s11033-011-11907, 2012.

Peng, D. Q., Jung, U. S., Lee, J. S., Kim, W. S., Jo, Y. H., Kim, M. J., Oh, Y. K., Baek, Y. C., Hwang, S. G., and Lee, H. G.: Effect of alcohol dehydrogenase 1C (ADH1C) genotype on vitamin A restriction and marbling in Korean native steers, Asian Austral. J. Anim., 30, 1099-1104, https://doi.org/10.5713/ajas.16.0708, 2017.
Suh, J. K., Lee, J. S., Kong, H., Lee, Y., and Lee, H. G.: The effect of single-nucleotide polymorphisms within heat shock protein beta 1 on beef quantity in Korean native steers, Arch. Anim. Breed., 63, 417-422, https://doi.org/10.5194/aab-63-417-2020, 2020.

Ward, A. K., McKinnon, J. J., Hendrick, S., and Buchanan, F. C.: The impact of vitamin A restriction and ADH1C genotype on marbling in feedlot steers, J. Anim. Sci., 90, 2476-2483, https://doi.org/10.2527/jas.2011-4404, 2012.

Zhang, Q., Lee, H. G., Kang, S. K., Baik, M. G., and Choi, Y. J.: Heat-shock protein beta 1 regulates androgenmediated bovine myogenesis, Biotechnol. Lett., 36, 1225-1231, https://doi.org/10.1007/s10529-014-1489-2, 2014.

Zhao, J., Xu, Z., Chen, A., You, X., Zhao, Y., He, W., Zhao, L., and Yang, S.: Identification of meat from yak and cattle using SNP markers with integrated allele-specific polymerase chain reaction-capillary electrophoresis method, Meat Sci., 148, 120 126, https://doi.org/10.1016/j.meatsci.2018.08.019, 2019. 